\title{
Construction of novel polyoxometalate/perylenediimide hybrid heterostructures for enhanced photocatalytic oxidation of mustard gas simulants
}

\author{
Meng-Hua $\mathrm{Li}^{\dagger}$, Shu-Li Lv ${ }^{\dagger}$, Ming-Hua You ${ }^{\dagger}$ „,* and Mei-Jin Lin ${ }^{\dagger, \#, \S, *}$
}

${ }^{\dagger}$ Key Laboratory of Molecule Synthesis and Function Discovery, College of Chemistry, Fuzhou University, China, 350116. E-mail: meijin_lin@fzu.edu.cn;

* College of Zhicheng, Fuzhou University, China, 350002

\# College of Materials Science and Engineering, Fuzhou University, China, 350116

$\S$ Fujian Provincial Key Laboratory of Electrochemical Energy Storage Materials, Fuzhou University, Fuzhou, Fujian, 350002, China

Table of Contents:

1. Syntheses of $\mathbf{P D I}, \mathbf{B r}_{2}$-PDI, $\mathbf{C l}_{\mathbf{4}}$-PDI $\quad$ S2

$\begin{array}{lll}\text { 2. Crystal data and structure refinement } & \text { S8 }\end{array}$

$\begin{array}{ll}\text { 3. Infrared spectral analyses } & \text { S9 }\end{array}$

4. Thermo-gravimetric analyses (TG) S10

5. X-ray powder diffraction analyses $\quad$ S11

6. The UV-vis diffuse reflection spectra $\quad$ S12

7. Kinetic analysis for photocatalytic oxidation CEES S13

8. Comparison of CEES Decomposition by Different Materials S14 


\section{Syntheses of PDI, $\mathrm{Br}_{2}$-PDI, $\mathrm{Cl}_{4}$-PDI}

All chemicals and solvents were purchased from commercial sources and used as supplied without further purification unless otherwise noted. 3,4,9,10-perylene tetracarboxylic dianhydride (98\%), 1,6,7,12-tetrachloroperylene-3,4,9,10-tetracarboxylic dianhydride [S1], 4-amino-3,5-di-methyl-pyrazole [S2], and 1,7-dibromo-perylene-3,4,9,10-tetracarboxylic dianhydride ${ }^{[\mathrm{S} 3]}$ were prepared according to the literatures. ethanol (analytical reagent grade), dichloromethane(analytical reagent grade), dimethylformamide (DMF, analytical reagentgrade), aceticacid (analytical reagent grade), propionicacid (analytical reagent grade), N-Methyl pyrrolidone (NMP,analytical reagent grade), hydrochloricacid $(\mathrm{HCl})$ and sulfuricacid were obtained from commercial suppliers. Ethylacetat (analytical reagent grade), iodine (analytical reagent grade), bromine(analytical reagent grade), anisole (analytical reagent grade), imidazole (analytical reagent grade). Nuclear magnetic resonance (NMR) spectra were recorded on a Bruker Avance 400 and 500 spectrometers with working frequencies of 400 and $500 \mathrm{MHz}$, respectively. Chemical shifts are given in parts per million (ppm) and referred to TMS as internal standard. ${ }^{1} \mathrm{H}$ coupling constants $\mathrm{J}$ are given in Hertz $(\mathrm{Hz})$. High-resolution mass spectra (HRMS) were acquired on the Thermo Scientific Executive plus Mass spectrometer equipped with electrospray ionization (ESI)source.

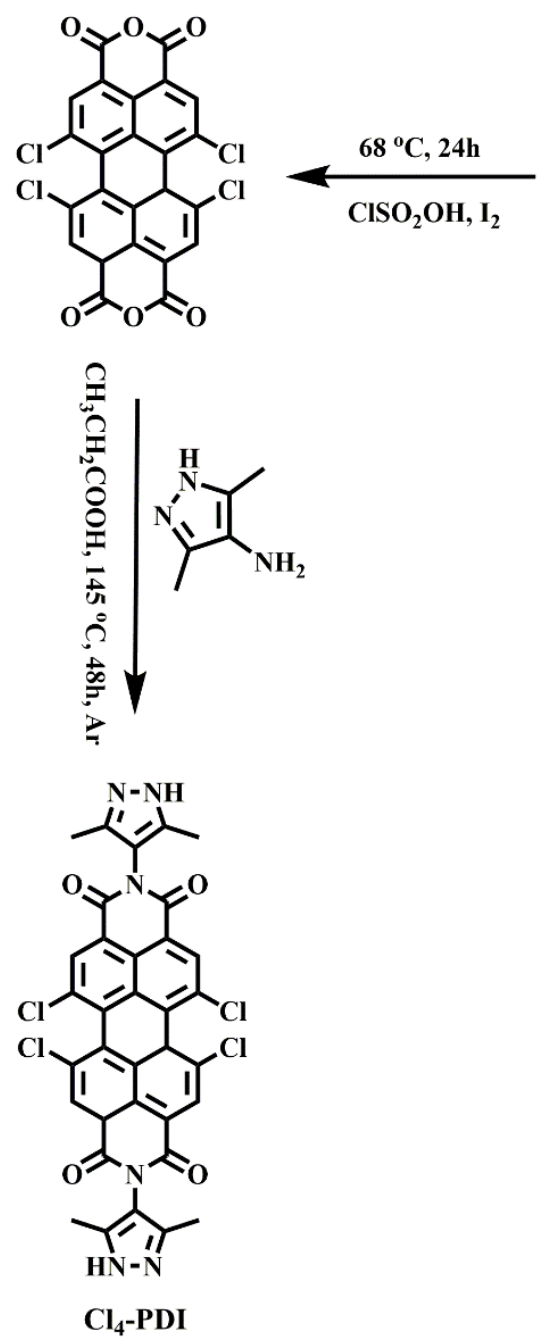

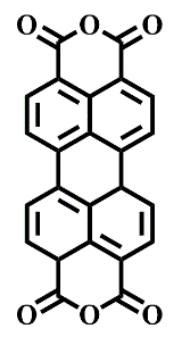

con. $\mathrm{H}_{2} \mathrm{SO}_{4}, 85^{\circ} \mathrm{C}$

$10 \mathrm{~h}, \mathrm{Br}_{2}, \mathrm{I}_{2}$

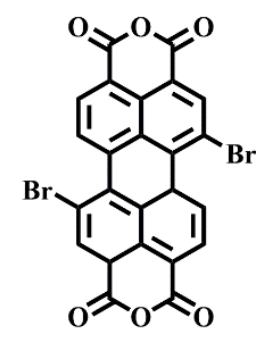
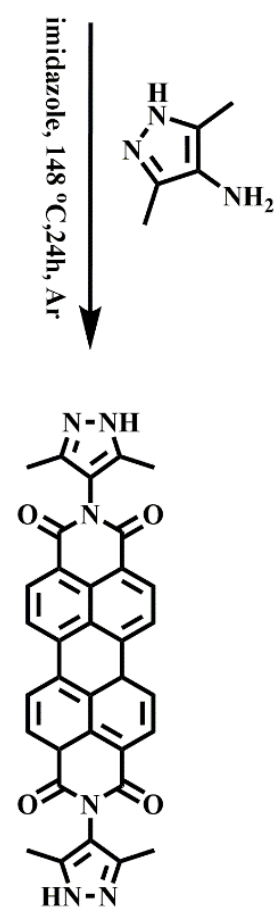

PDI
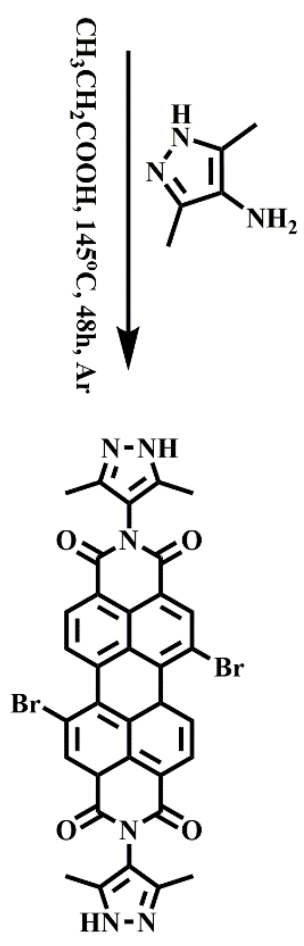

$\mathrm{Br}_{2}$-PDI 
Scheme S1. Synthesis route for precursors $\mathbf{P D I}, \mathbf{B r}_{2}-\mathrm{PDI}, \mathbf{C l}_{4}-\mathrm{PDI}$.

Synthesis of N,N-bis(3,5-dimethyl-1H-pyrazole-4-yl)-perylene-3,4,9,10-bis(dicarboximide) (PDI): 4-Amino-3,5-dimethylpyrazole $(2.096 \mathrm{~g}, 18.80 \mathrm{mmol})$ was added to a mixture of 1,6,7,12-tetrachloroperylene-3,4,9,10-tetracarboxylic dianhydride (1.992 g, $3.76 \mathrm{mmol})$ and imidazole (20 $\mathrm{mg}$ ) under argon atmosphere. The reaction mixture was stirred and heated at $148{ }^{\circ} \mathrm{C}$ for $24 \mathrm{~h}$. Then the reaction was quenched with $400 \mathrm{~mL}$ of $2 \mathrm{M}$ hydrochloric acid and stired for $4 \mathrm{~h}$. The resultant precipitate was filtered and washed with deionized water until the filtrate became neutral. The red crude productwas purified by silica gel column chromatography with methanol and dichloromethane(DCM) (from 1:1 to 10:1 v/v) to give product PDI as a red solid $1.213 \mathrm{~g}\left(2.09 \mathrm{mmol}, 55.6 \%\right.$ yield). ${ }^{1} \mathrm{H} \mathrm{NMR}\left(400 \mathrm{MHz}, \mathrm{CF}_{3} \mathrm{COOD}\right) \delta 8.97$ (s, $8 \mathrm{H}), 2.41(\mathrm{~s}, 12 \mathrm{H}) ;{ }^{13} \mathrm{C} \mathrm{NMR}\left(100 \mathrm{MHz}, \mathrm{CF}_{3} \mathrm{COOD}\right) \delta 164.84,145.95,137.02,134.07,132.51,130.33$, $127.05,124.87,121.83,8.32$; MS(ESI-MS) m/z: Calculated for $\mathrm{C}_{34} \mathrm{H}_{24} \mathrm{~N}_{6} \mathrm{O}_{4} 580.1854$, Found: 580.1820 。

Synthesis of N,N-bis(3,5-dimethyl-1H-pyrazole-4-yl)-1,7-dibromoperylene-3,4,9,10-tetracarboxylic diimide (Br 2 -PDI): 4-Amino-3,5-dimethylpyrazole (3.600 g, $32.45 \mathrm{mmol})$ was added to a mixture of 1,7-dibromo-perylene-3,4,9,10-tetracarboxylic dianhydride (4.520 g, $8.00 \mathrm{mmol})$ and $180 \mathrm{ml}$ propionic acid under argon atmosphere. The reaction mixture was stirred and heated at $145{ }^{\circ} \mathrm{C}$ for $48 \mathrm{~h}$. After cooling to room temperature, the reaction was quenched with $400 \mathrm{~mL}$ of deionized water and stired for $2 \mathrm{~h}$. The resultant precipitate was filtered and washed with saturated $\mathrm{NaHCO}_{3}$ until the filtrate became neutral. The red crude productwas purified by silica gel column chromatography with methanol and dichloromethane (DCM) (from 1:100 to 5:100 v/v) to give product $\mathbf{B r}_{2}$-PDI as a red solid $3.710 \mathrm{~g}\left(5.01 \mathrm{mmol}, 62.8 \%\right.$ yield). ${ }^{1} \mathrm{H}$ NMR (400 $\left.\mathrm{MHz}, \mathrm{CDCl}_{3}\right) \delta 9.59(\mathrm{~d}, \mathrm{~J}=8.5 \mathrm{~Hz}, 2 \mathrm{H}), 9.04(\mathrm{~s}, 2 \mathrm{H}), 8.83(\mathrm{~d}, \mathrm{~J}=7.5 \mathrm{~Hz}, 2 \mathrm{H}), 2.21(\mathrm{~s}, 12 \mathrm{H}) ;{ }^{13} \mathrm{C}$ NMR $(100$ $\left.\mathrm{MHz}, \mathrm{CF}_{3} \mathrm{COOD}\right) \delta 154.16,145.87,140.23,135.31,135.10,132.25,129.73,129.58,127.70,122.00,121.75$, 121.24, 99.98, 8.43, 8.31; MS(ESI-MS) m/z: Calculated for $\mathrm{C}_{34} \mathrm{H}_{22} \mathrm{Br}_{2} \mathrm{~N}_{6} \mathrm{O}_{4} 738.0043$, Found: 738.0005 .

Synthesis of N,N'-bis(3,5-dimethyl-1H-pyrazole-4-yl)-1,6,7,12-tetrachloroperylene-3,4,9,10-tetracarboxy diimide $\left(\mathbf{C l}_{4}\right.$-PDI) : The synthetic procedure for $\mathbf{C l}_{4}$-PDI was similar to that used for $\mathbf{B r}_{2}-\mathbf{P D I}$, with 1,6,7,12-tetrachloroperylene-3,4,9,10-tetracarboxylic dianhydride $(8.45 \mathrm{mmol}, 4.500 \mathrm{~g})$ instead of 1,7-dibromo-perylene-3,4,9,10-tetracarboxylic dianhydride. A red solid product was obtained $5.110 \mathrm{~g}$ (7.16 mmol, 84.7\% yield) ${ }^{1} \mathrm{H}$ NMR (400 MHz, DMSO-d 6 ) $\delta 12.50(\mathrm{~s}, 2 \mathrm{H}), 8.64(\mathrm{~s}, 4 \mathrm{H}), 2.05(\mathrm{~s}, 12 \mathrm{H}) ;{ }^{13} \mathrm{C}$ NMR (100 MHz, $\left.\mathrm{CF}_{3} \mathrm{COOD}\right) \delta 163.40,145.80,136.91,134.76,131.58,130.62$, 123.81, 121.76, 115.12, 8.27 ppm; MS(ESI-MS) m/z: Calculated for $\mathrm{C}_{34} \mathrm{H}_{18} \mathrm{Cl}_{4} \mathrm{~N}_{6} \mathrm{O}_{4}$ 714.0138, Found: 714.0163 . 


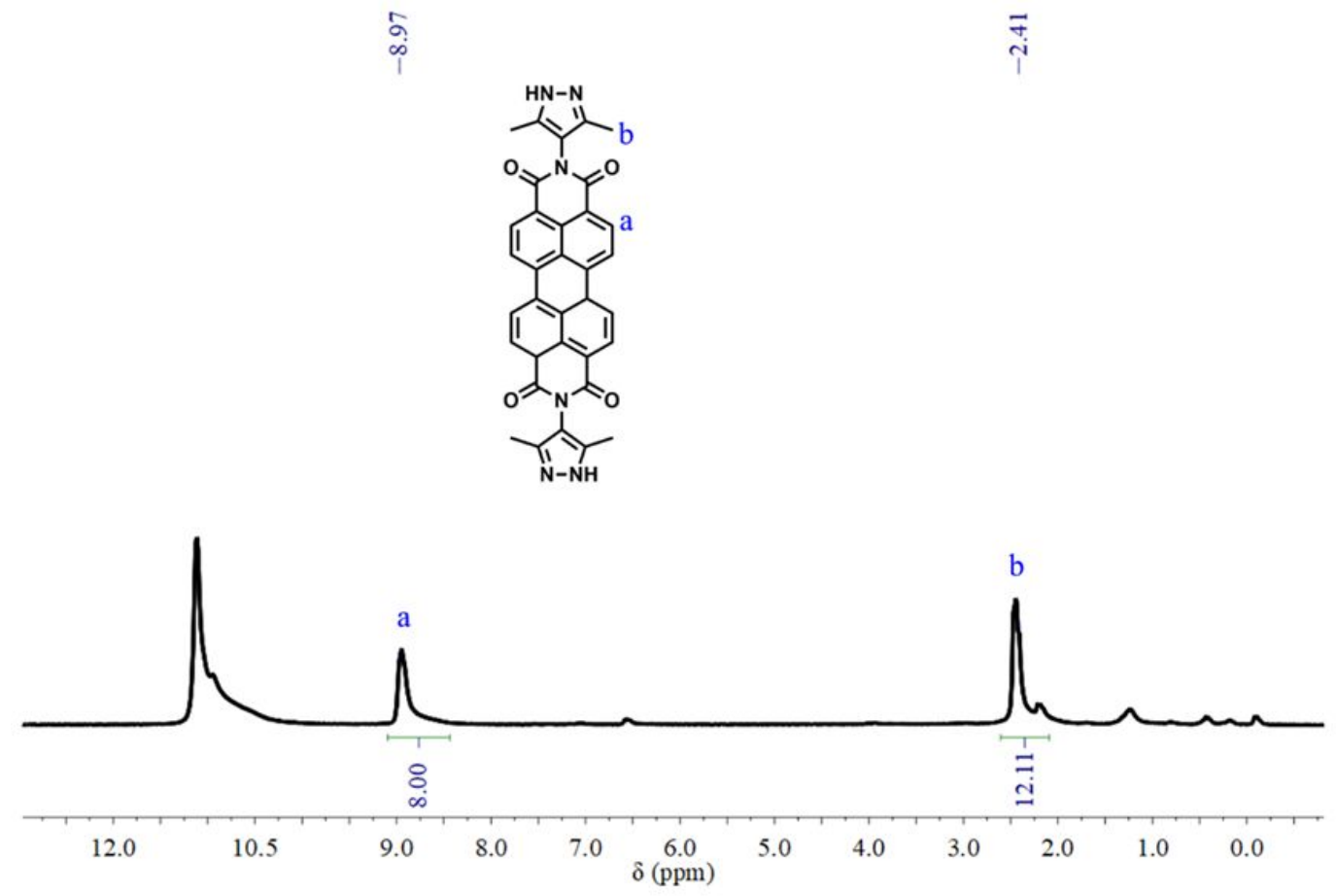

$\underset{\text { i }}{\text { i }}$

Figure S1 ${ }^{1} \mathrm{H}$ NMR spectrum of PDI (400 MHz, $\left.\mathrm{CF}_{3} \mathrm{COOD}, 298 \mathrm{~K}\right)$.

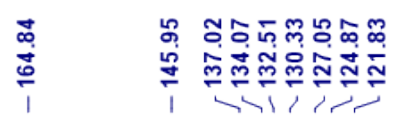

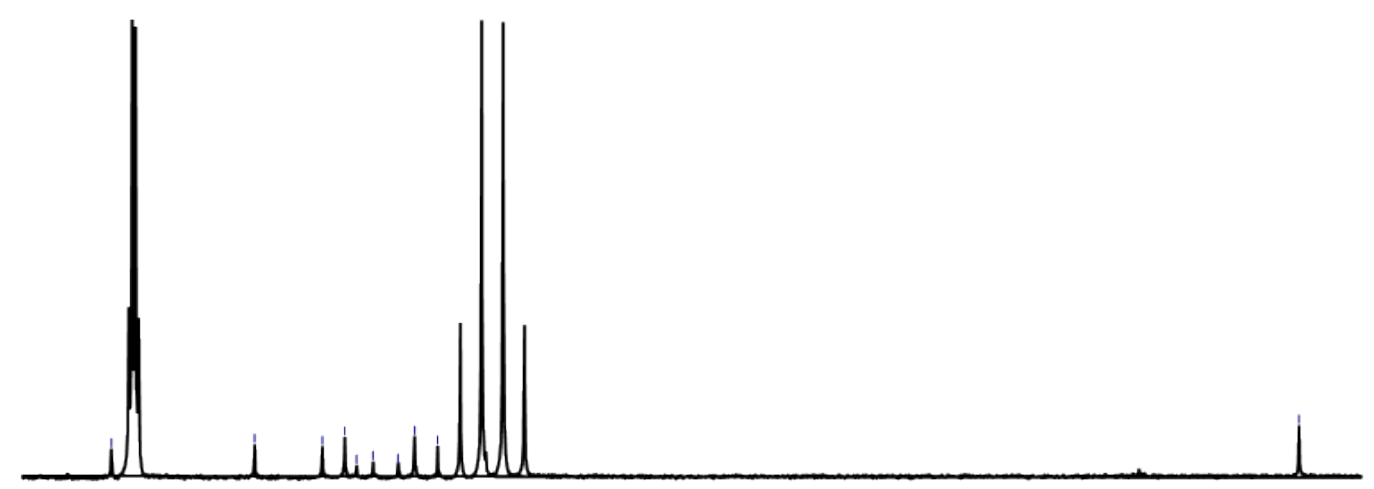

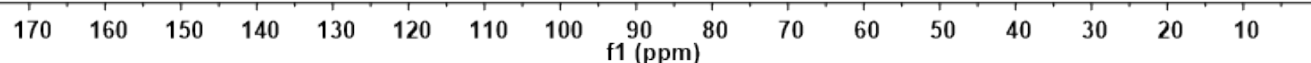

Figure S2 ${ }^{13} \mathrm{C}$ NMR spectrum of PDI (100 MHz, $\left.\mathrm{CF}_{3} \mathrm{COOD}, 298 \mathrm{~K}\right)$. 


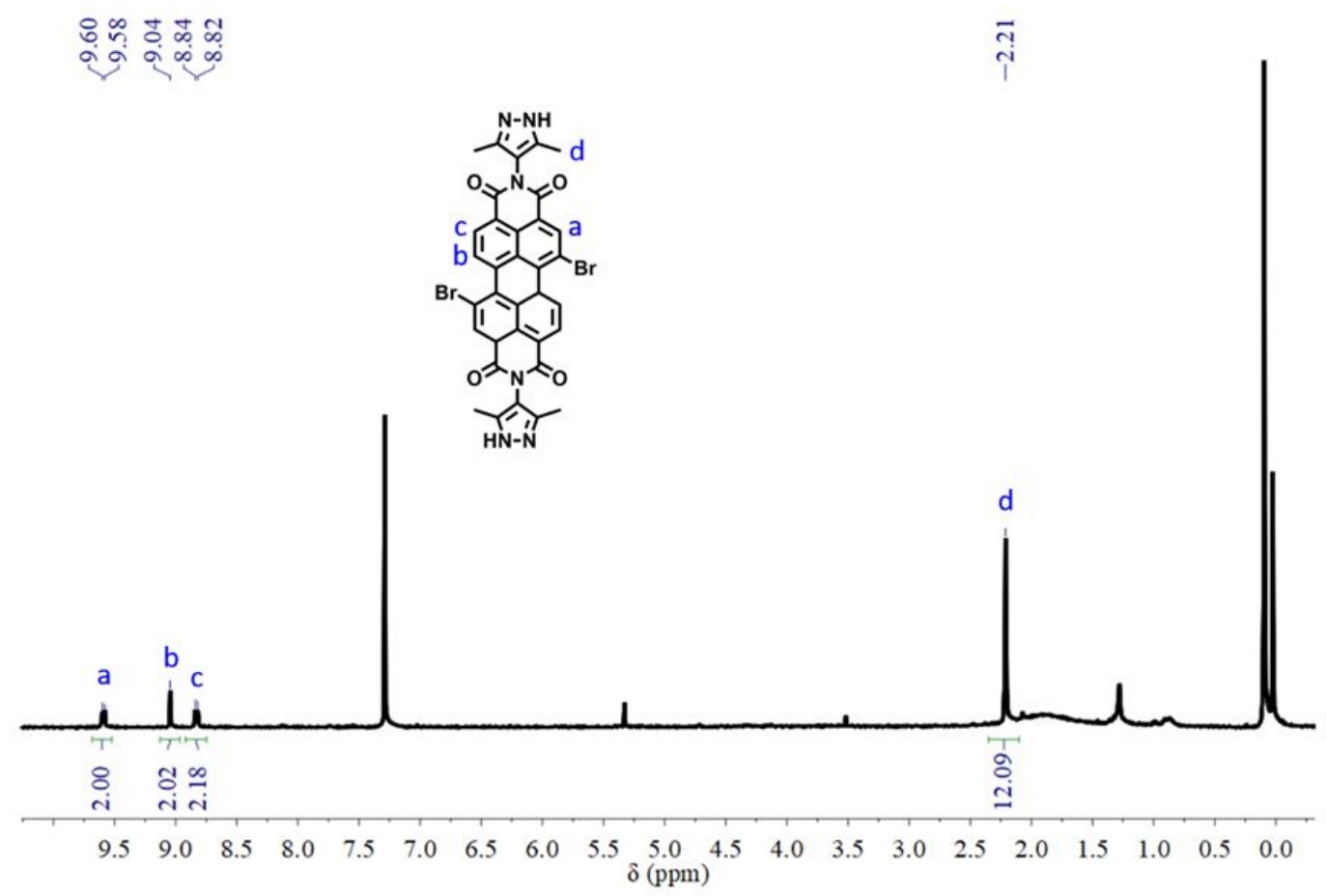

Figure S3 ${ }^{1} \mathrm{H}$ NMR spectrum of $\mathbf{B r}_{2}$-PDI (400 MHz, $\mathrm{CDCl}_{3}, 298 \mathrm{~K}$ ).

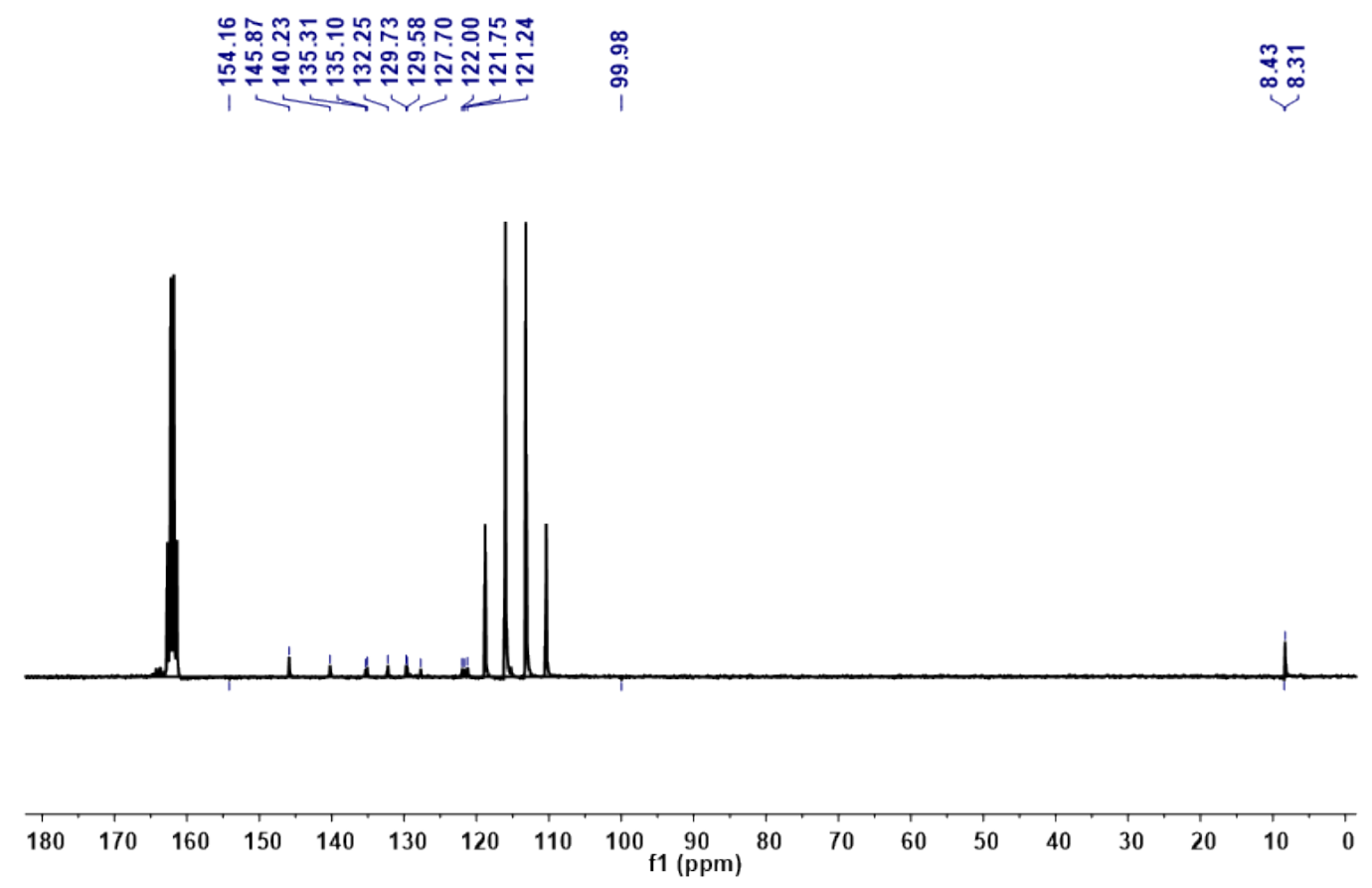

Figure $\mathbf{S} 4{ }^{13} \mathrm{C}$ NMR spectrum of $\mathbf{B r}_{2}$-PDI (100 MHz, $\left.\mathrm{CF}_{3} \mathrm{COOD}, 298 \mathrm{~K}\right)$. 


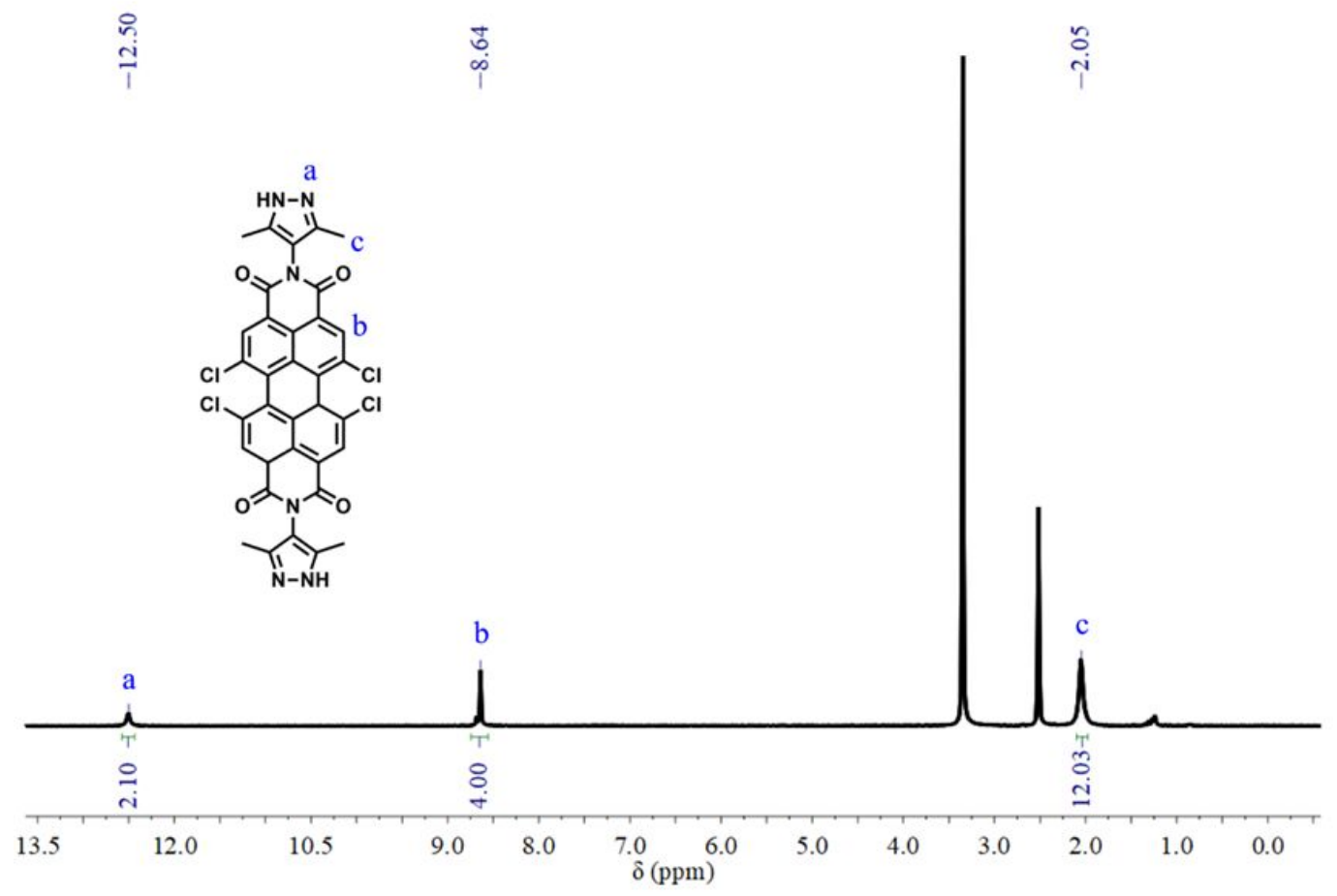

Figure S5 ${ }^{1} \mathrm{H}$ NMR spectrum of $\mathbf{C l}_{4}$-PDI (400 MHz, $\mathrm{CDCl}_{3}, 298 \mathrm{~K}$ ).
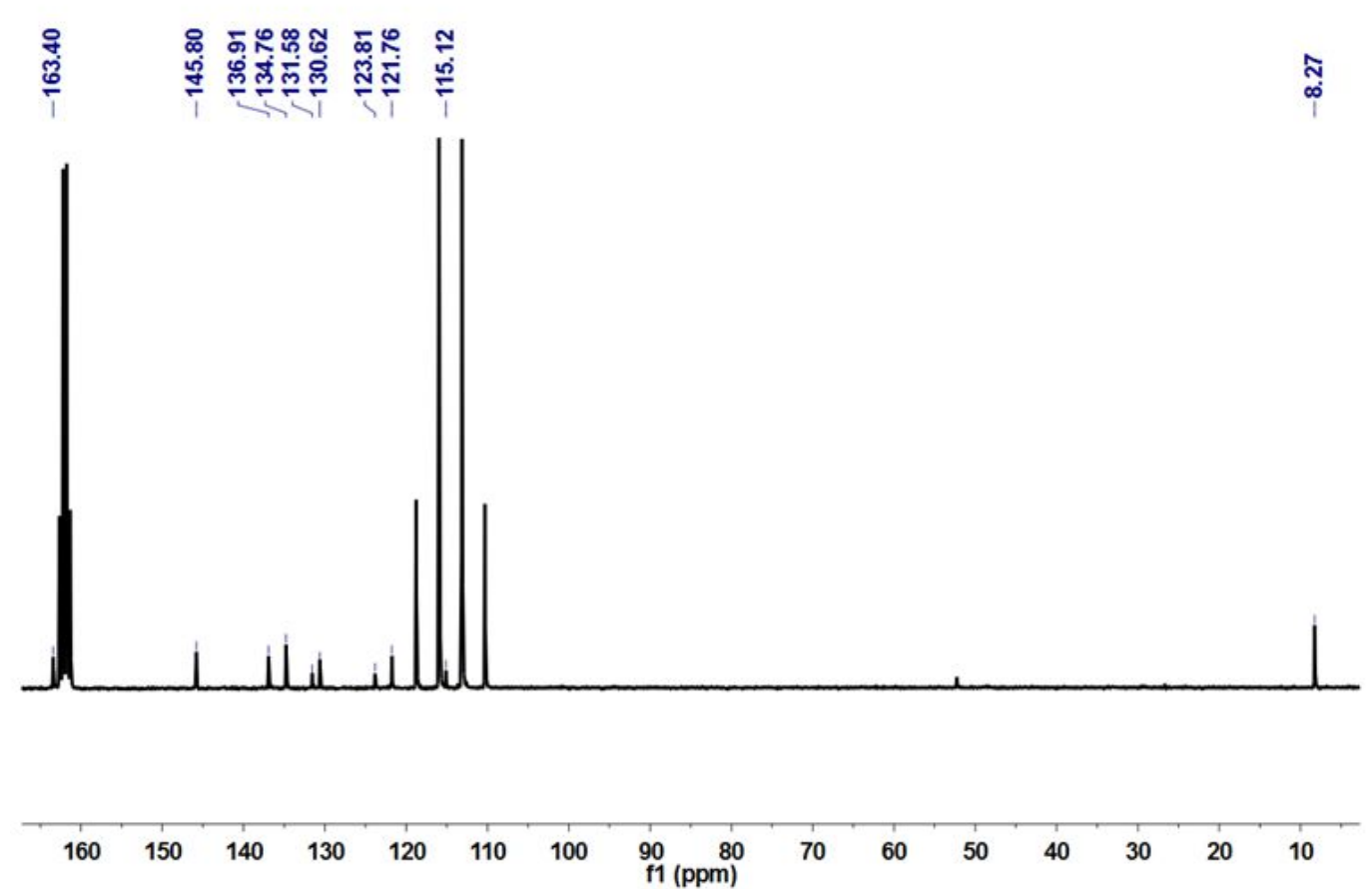

Figure S6 ${ }^{13} \mathrm{C}$ NMR spectrum of $\mathbf{C l}_{\mathbf{4}}$-PDI (100 MHz, $\left.\mathrm{CF}_{3} \mathrm{COOD}, 298 \mathrm{~K}\right)$. 


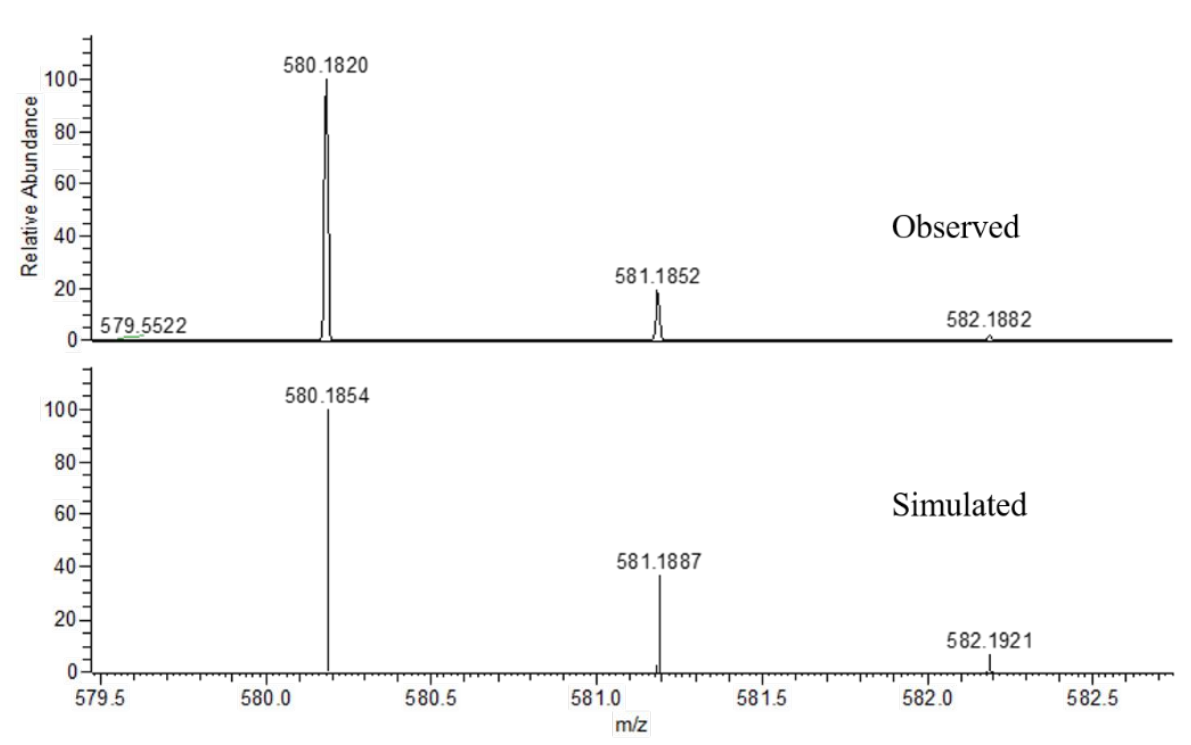

Figure S7 ESI-MS (positive mode) of PDI with the observed and simulated isotope patterns.

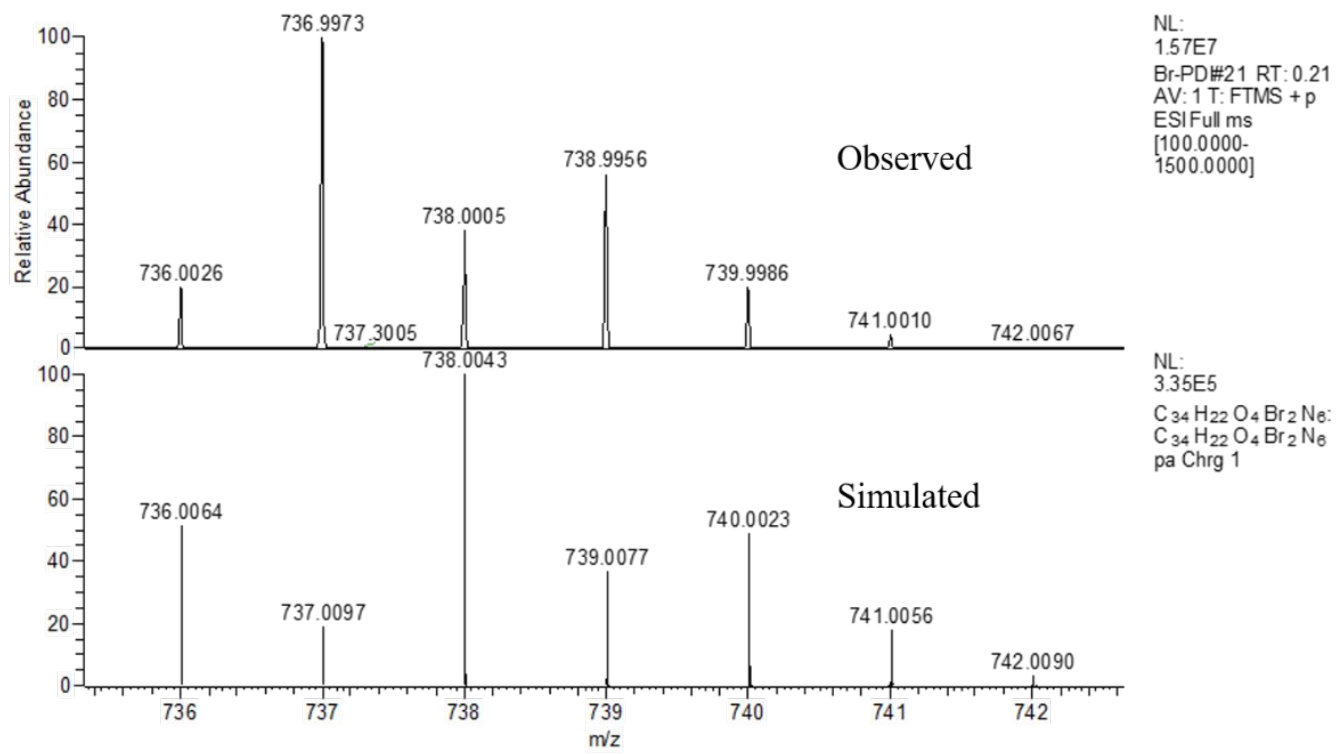

Figure S7 ESI-MS (positive mode) of $\mathbf{B r}_{2}$-PDI with the observed and simulated isotope patterns. 


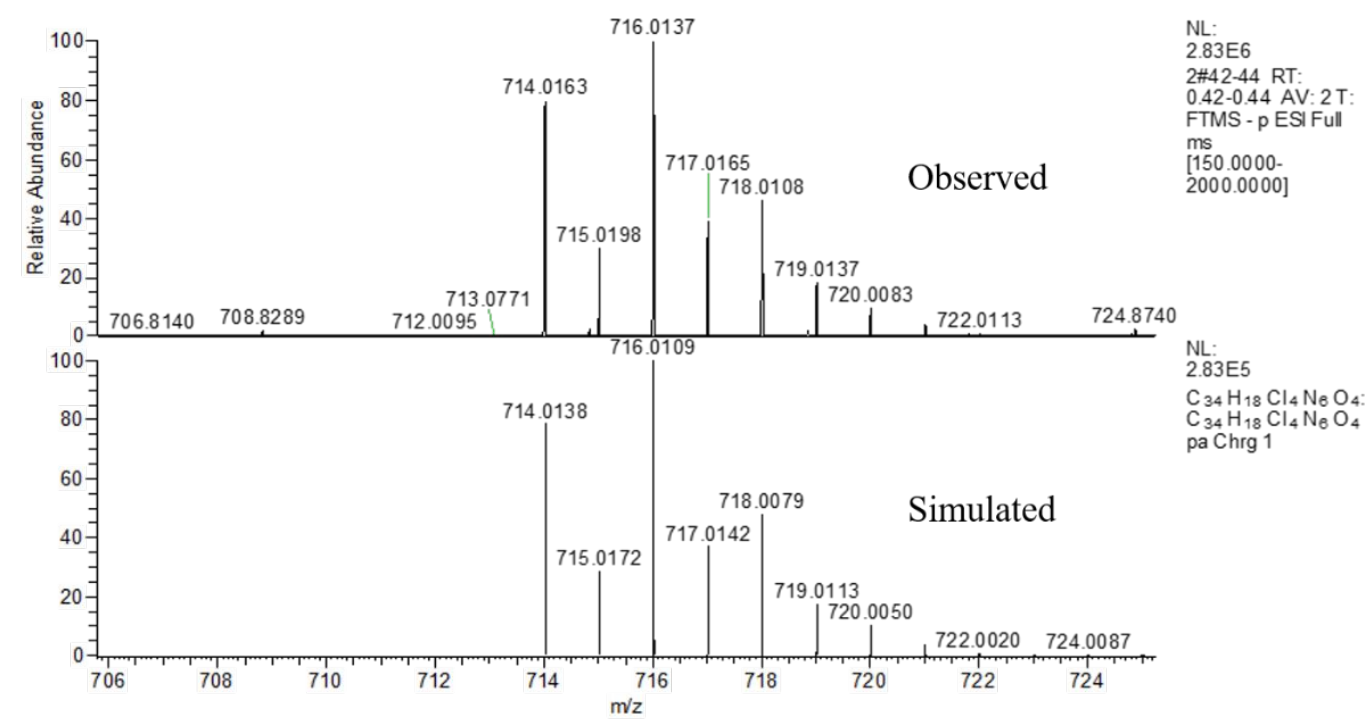

Figure S7 ESI-MS (positive mode) of $\mathbf{C l}_{4}$-PDI with the observed and simulated isotope patterns.

\section{Crystal data and structure refinement for hybrid 1-3}

Table 1. Crystallographic Data and Structural Refinements Parameters for hybrid 1-3.

\begin{tabular}{|c|c|c|c|}
\hline hybrid & 1 & 2 & 3 \\
\hline Empirical formula & $\mathrm{C}_{38} \mathrm{H}_{32} \mathrm{~N}_{6} \mathrm{O}_{24} \mathrm{Si}_{0.5} \mathrm{~W}_{6}$ & $\mathrm{C}_{38} \mathrm{H}_{28} \mathrm{Br}_{2} \mathrm{~N}_{6} \mathrm{O}_{30.67} \mathrm{Si}_{0.67} \mathrm{~W}_{8}$ & $\mathrm{C}_{38} \mathrm{H}_{28} \mathrm{Cl}_{4} \mathrm{~N}_{6} \mathrm{O}_{24} \mathrm{Si}_{0.5} \mathrm{~W}_{6}$ \\
\hline Formula weight & 2073.84 & 2708.68 & 2211.61 \\
\hline Temperature/K & 296.15 & 296.15 & $286(4)$ \\
\hline Crystal system & triclinic & orthorhombic & triclinic \\
\hline Space group & $P-1$ & Pbcn & $P-1$ \\
\hline $\mathrm{a} / \AA$ & $12.1137(9)$ & $29.4600(6)$ & $14.1417(10)$ \\
\hline $\mathrm{b} / \AA$ & $13.8542(11)$ & $27.7833(6)$ & $14.3230(10)$ \\
\hline $\mathrm{c} / \AA$ & $16.8371(14)$ & $22.2931(4)$ & $14.7165(11)$ \\
\hline$\alpha /^{\circ}$ & $113.833(3)$ & 90 & $65.521(7)$ \\
\hline$\beta /{ }^{\circ}$ & $97.159(3)$ & 90 & $79.893(6)$ \\
\hline$\gamma /{ }^{\circ}$ & $96.209(3)$ & 90 & $78.211(6)$ \\
\hline Volume $/ \AA^{3}$ & $2525.2(4)$ & $18246.8(6)$ & $2641.9(4)$ \\
\hline $\mathrm{Z}$ & 2 & 12 & 2 \\
\hline$\rho_{\text {calc }} \mathrm{g} / \mathrm{cm}^{3}$ & 2.727 & 2.957 & 2.780 \\
\hline$\mu / \mathrm{mm}^{-1}$ & 13.711 & 16.479 & 13.310 \\
\hline $2 \Theta$ range for data collection $/{ }^{\circ}$ & 4.67 to 50.054 & 4.172 to 50.088 & 5.674 to 50.052 \\
\hline Reflections collected & 56741 & 161287 & 38265 \\
\hline Independent reflections & 8917 & 16125 & 9313 \\
\hline Data/restraints/parameters & $8917 / 114 / 699$ & $16125 / 18 / 1197$ & $9313 / 195 / 735$ \\
\hline Goodness-of-fit on $\mathrm{F}^{2}$ & 1.011 & 1.052 & 1.024 \\
\hline Final $R$ indexes $[I>=2 \sigma(I)]$ & $\begin{aligned} \mathrm{R}_{1} & =0.0440 \\
\mathrm{wR}_{2} & =0.0864\end{aligned}$ & $\begin{array}{c}\mathrm{R}_{1}=0.0649 \\
\mathrm{wR}_{2}=0.1592\end{array}$ & $\begin{array}{c}\mathrm{R}_{1}=0.0607 \\
\mathrm{wR}_{2}=0.1336\end{array}$ \\
\hline Final $\mathrm{R}$ indexes [all data] & $\begin{array}{c}\mathrm{R}_{1}=0.0717 \\
\mathrm{wR}_{2}=0.1012\end{array}$ & $\begin{array}{c}\mathrm{R}_{1}=0.0736 \\
\mathrm{wR}_{2}=0.1668\end{array}$ & $\begin{array}{c}\mathrm{R}_{1}=0.1237 \\
\mathrm{wR}_{2}=0.1685\end{array}$ \\
\hline Largest diff. peak/hole / e $\AA^{-3}$ & $2.02 /-1.80$ & $5.30 /-4.31$ & $2.43 /-1.34$ \\
\hline
\end{tabular}




\section{Infrared spectral analyses}
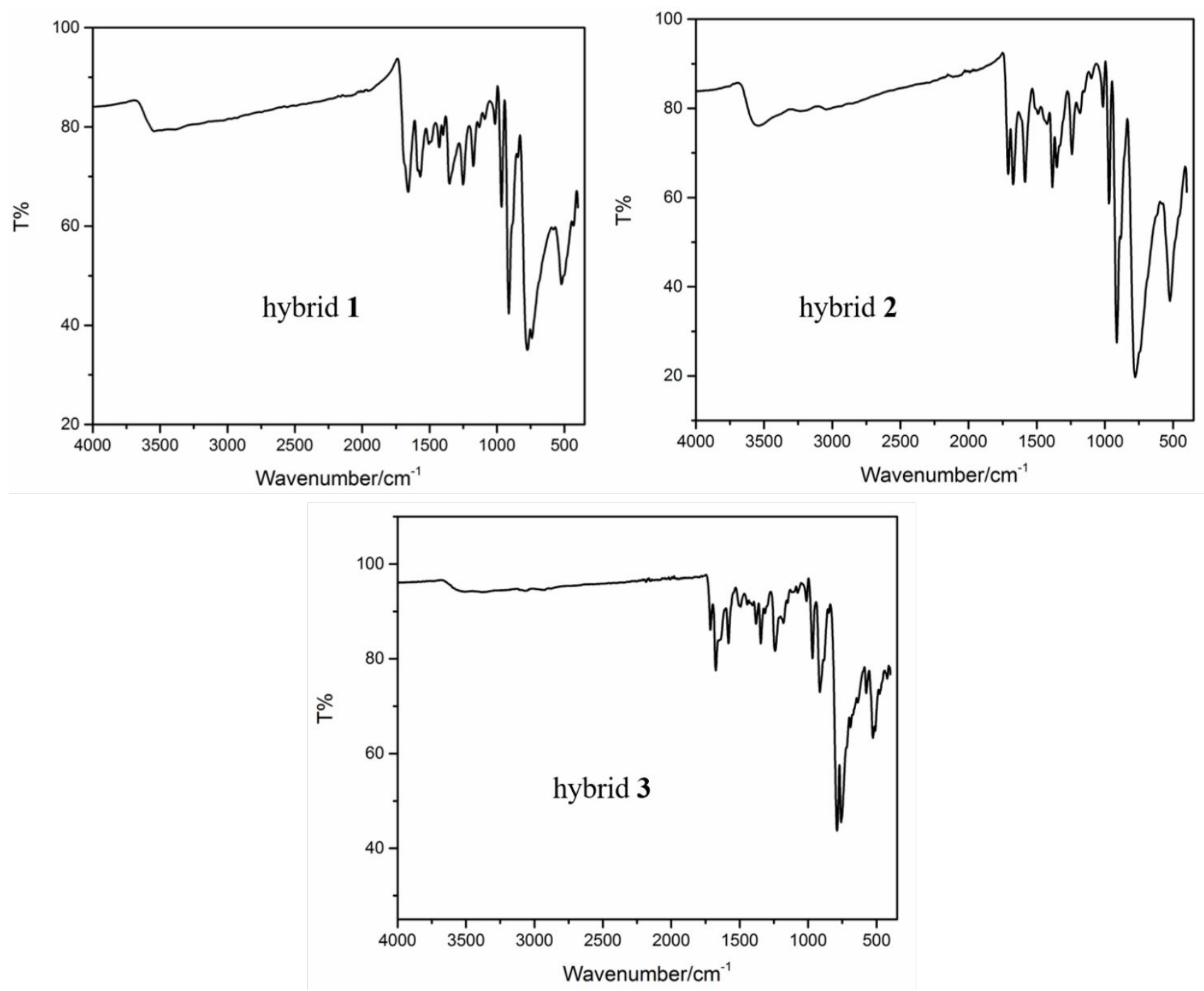

Figure S8. Infrared spectra of hybrids 1-3. 
4. Thermo-gravimetric analyses (TG)

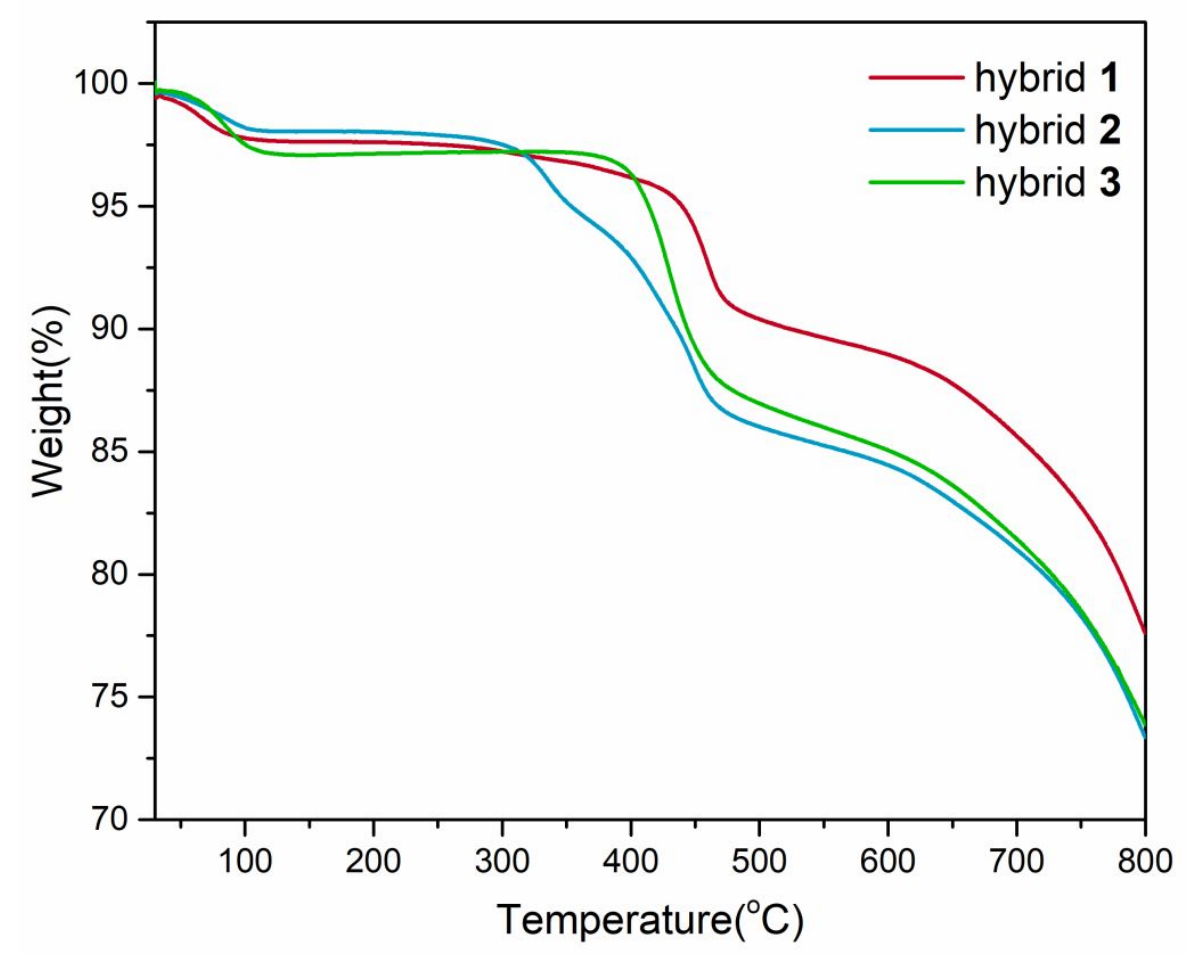

Figure S9. The TG curve of hybrids $\mathbf{1 - 3}$ under $\mathrm{N}_{2}$ atmosphere with a heating rate of $10{ }^{\circ} \mathrm{C} / \mathrm{min}$. 
5. X-ray powder diffraction analyses
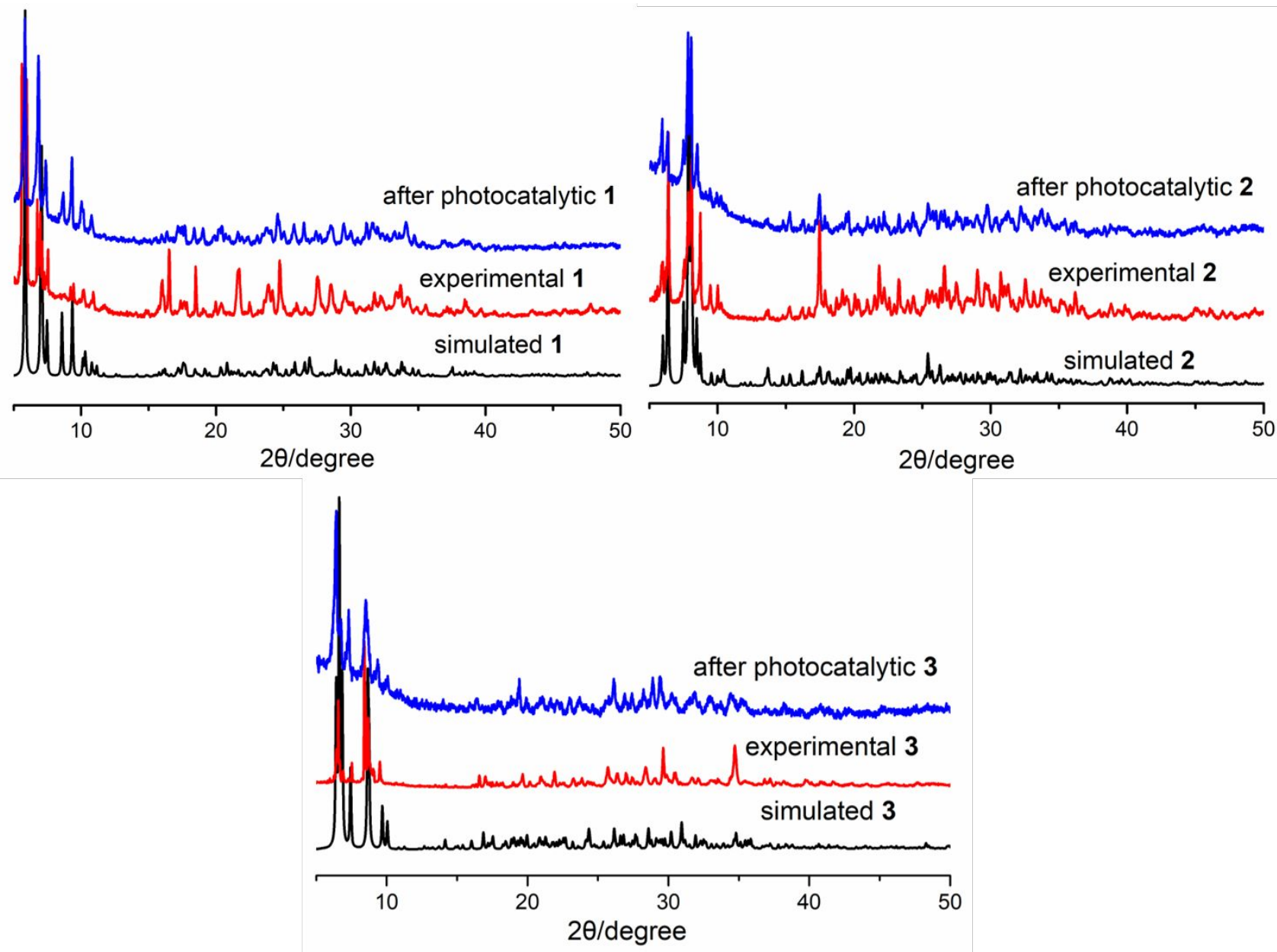

Figure S10. PXRD patterns of compounds 1-3 after irradiation. 
6. The UV-vis diffuse reflection spectra

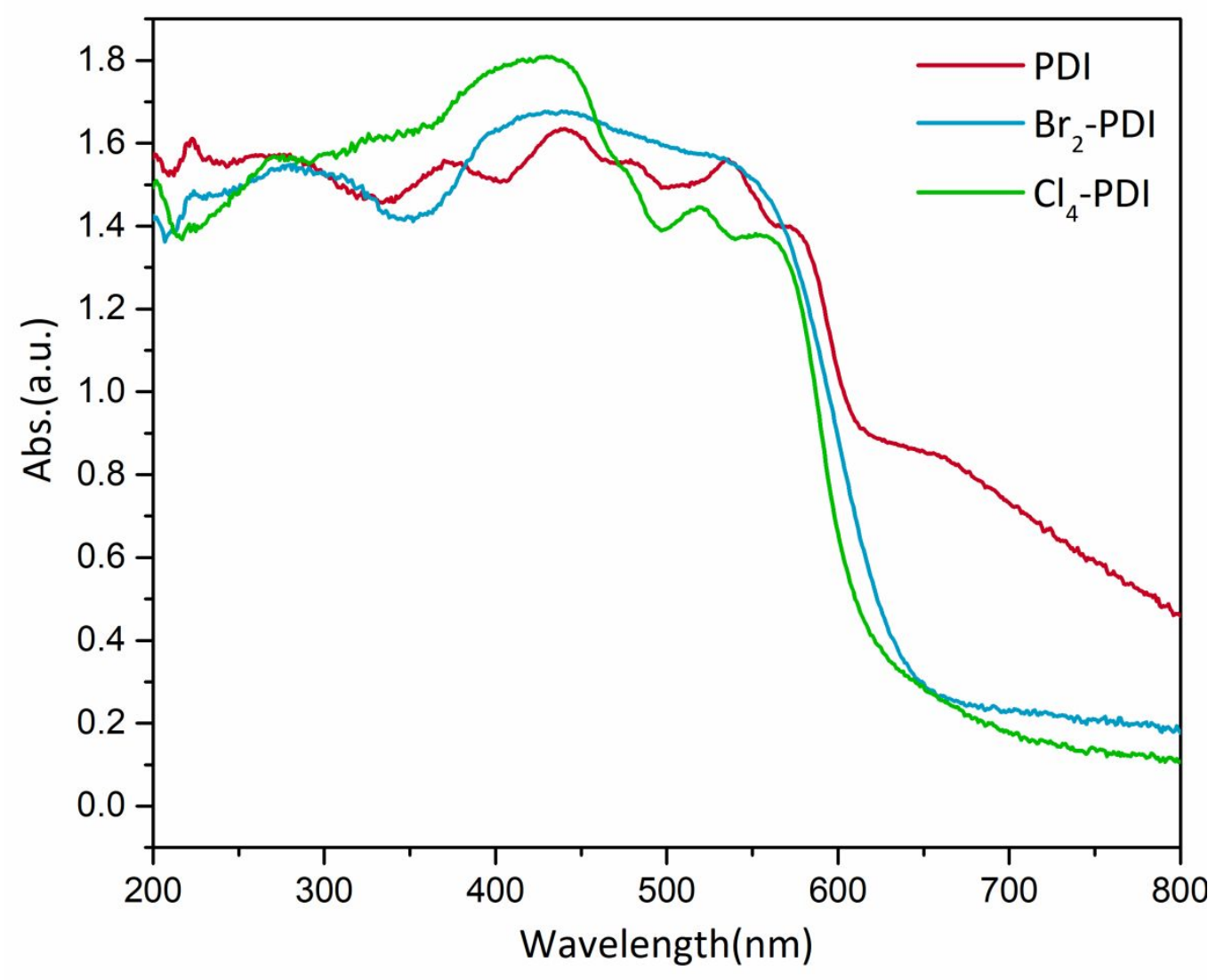

Figure S11. The UV-vis diffuse reflection spectra (DRS) of PDI, $\mathbf{B r}_{2}-\mathbf{P D I}$, and $\mathbf{C l}_{4}-\mathbf{P D I}$. 
7. Kinetic analysis for photocatalytic oxidation CEES

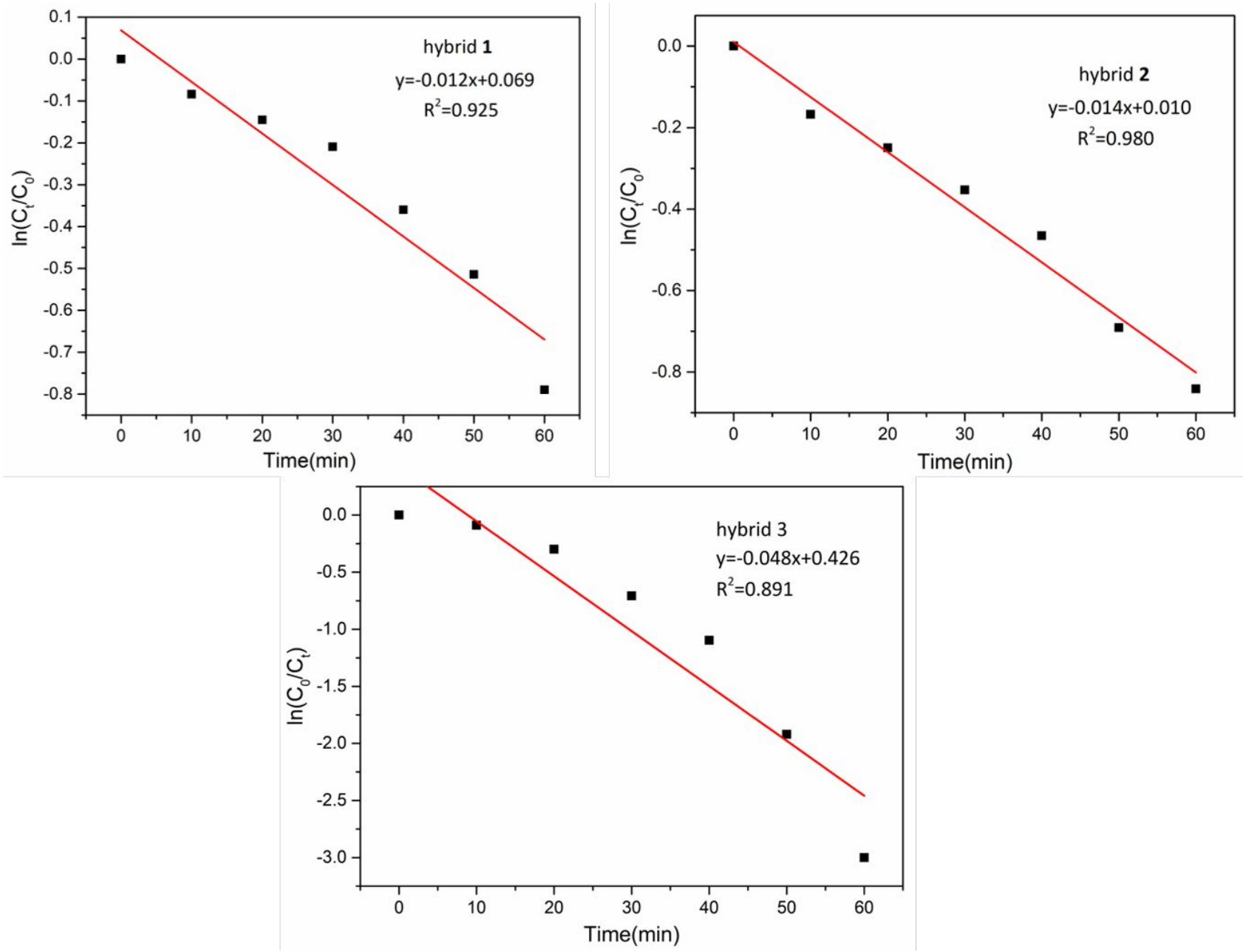

Figure S12. Kinetic analysis of CEES oxidation for hybrids 1-3. 


\begin{tabular}{|c|c|c|c|c|c|c|}
\hline catalyst & $\begin{array}{c}\text { effective } \\
\text { photosensitizer }\end{array}$ & atmosphere & $\begin{array}{l}\text { time } \\
(\min )\end{array}$ & $\begin{array}{c}\text { conversion } \\
(\%)\end{array}$ & $\begin{array}{c}\text { selectivity } \\
(\%)\end{array}$ & refs \\
\hline PCN-222/ & porphyrin & $\mathrm{O}_{2}$ (blue LED) & 25 & 93 & 100 & S4 \\
\hline NU-1000 & pyrene & $\mathrm{O}_{2}(\mathrm{UV}$ LED) & 15 & 100 & 100 & S5 \\
\hline Por-MOFs & porphyrin & $\mathrm{O}_{2}$ (blue LED) & 60 & 100 & 100 & S6 \\
\hline UiO-68-TBTD & TBTD & air(blue LED) & 10 & 100 & 100 & S7 \\
\hline IBDP-POP & BODIPY & $\mathrm{O}_{2}$ (green LED) & 30 & 100 & 100 & S8 \\
\hline Compound 1 & $\mathrm{Me}_{4}$-PDI & $\mathrm{O}_{2}$ (light) & 60 & 83.8 & 99 & This work \\
\hline Compound 2 & $\mathrm{Me}_{4}-\mathrm{Br}_{2}$-PDI & $\mathrm{O}_{2}$ (light) & 60 & 85.6 & 99 & This work \\
\hline Compound $\mathbf{3}$ & $\mathrm{Me}_{4}-\mathrm{Cl}_{4}$-PDI & $\mathrm{O}_{2}$ (light) & 60 & 99.9 & 99 & This work \\
\hline
\end{tabular}

8. Table 2. Comparison of CEES Decomposition by Different Materials in Recent Years 


\section{References}

S1 Gong, H. X.; Cao, Z.; Li, M. H.; Liao, S. H.; Lin, M. J. Photoexcited perylene diimide radical anions for the reduction of aryl halides: a bay-substituent effect. Organic Chemistry Frontiers 2018, 5 (15), 2296-2302.

S2 Ke, H.; Weng, L. J.; Chen, S. Y.; Chen, J.-Z.; Lin, M. J. Naphthalene diimide cocrystals: A facile approach to tune the optical properties. Dyes and Pigments 2015, 113, 318-324.

S3 Wurthner, F.; Stepanenko, V.; Chen, Z.; Saha-Moller, C. R.; Kocher, N.; Stalke, D. Preparation and characterization of regioisomerically pure 1,7-disubstituted perylene bisimide dyes. J Org Chem 2004, 69 (23), 7933-7939.

S4 Liu, Y.; Howarth, A. J.; Hupp, J. T.; Farha, O. K., Selective Photooxidation of a Mustard-Gas Simulant Catalyzed by a Porphyrinic Metal-Organic Framework. Angew Chem 2015, 54, (31), 9001-9005.

S5 Liu, Y.; Buru, C. T.; Howarth, A. J.; Mahle, J. J.; Buchanan, J. H.; DeCoste, J. B.; Hupp, J. T.; Farha, O. K., Efficient and selective oxidation of sulfur mustard using singlet oxygen generated by a pyrene-based metal-organic framework. J Mater Chem A 2016, 4, (36), 13809-13813.

S6 Pereira, C. F.; Liu, Y.; Howarth, A.; Figueira, F.; Rocha, J.; Hupp, J. T.; Farha, O. K.; Tomé, J. P. C.; Almeida Paz, F. A., Detoxification of a Mustard-Gas Simulant by Nanosized Porphyrin-Based Metal-Organic Frameworks. ACS Applied Nano Materials 2018, 2, (1), 465-469.

S7 Zhang, W. Q.; Cheng, K.; Zhang, H.; Li, Q. Y.; Ma, Z.; Wang, Z.; Sheng, J.; Li, Y.; Zhao, X.; Wang, X. J., Highly Efficient and Selective Photooxidation of Sulfur Mustard Simulant by a Triazolobenzothiadiazole-Moiety-Functionalized Metal-Organic Framework in Air. Inorg Chem 2018, 57, (8), 4230-4233.

S8 Atilgan, A.; Cetin, M. M.; Yu, J.; Beldjoudi, Y.; Liu, J.; Stern, C. L.; Cetin, F. M.; Islamoglu, T.; Farha, O. K.; Deria, P.; Stoddart, J. F.; Hupp, J. T., Post-Synthetically Elaborated BODIPY-Based Porous Organic Polymers (POPs) for the Photochemical Detoxification of a Sulfur Mustard Simulant. J Am Chem Soc 2020, 142, (43), 18554-18564. 